\title{
On the Nonlinear Response of a Flexible Connecting Rod
}

In two recent papers (Chen, J.-S., and Chen, K.-L., 2001, "The Role of Lagrangian Strain in the Dynamic Response of a Flexible Connecting Rod," ASME Journal of Mechanical Design, 123, pp. 542-548; Chen, J.-S., and Huang, C.-L., 2001, "Dynamic Analysis of Flexible Slider-Crank Mechanisms With Nonlinear Finite Element Method," Journal of Sound and Vibration, 246, pp. 389-402) we reported that previous researches of others on the dynamic response of a flexible connecting rod may have overestimated the deflections by ten folds when the crank rotates near the bending natural frequency of the connecting rod because terms of significant order of magnitude were ignored inadequately. While the findings in (Chen, J.-S., and Chen, K.-L., 2001, "The Role of Lagrangian Strain in the Dynamic Response of a Flexible Connecting Rod," ASME Journal of Mechanical Design, 123, pp. 542-548; Chen, J.-S., and Huang, C.-L., 2001, "Dynamic Analysis of Flexible Slider-Crank Mechanisms With Nonlinear Finite Element Method," Journal of Sound and Vibration, 246, pp. 389-402.) were obtained via numerical simulations, the present paper emphasizes the analytical approach with an aim to exploring the physical insights behind these numerical results. The equations of motion are first derived by applying Hamilton's principle with all high order terms in the strain energy function being retained. After careful examination of the order of magnitude of each term, the coupled equations are simplified to a single one in terms of the transverse deflection, which turns out to be a Duffing equation under parametric and external excitations simultaneously. Closed-form approximations of the dynamic response are then derived by using multiple scale method. It is found that the combined effects of parametric and external excitations dominate the response when $\Omega$ is close to 0.5 and 1 . Away from these two speed ranges, on the other hand, the response is dominated by the external excitation alone. [DOI: 10.1115/1.1631571]

\section{Introduction}

The flexible connecting rod of a slider-crank mechanism can be considered as a beam undergoing flexible deformation superposed on large rigid body motion and may deform in both the axial and transverse directions. A complete formulation must take into account the coupling effect of the axial and transverse vibrations. However, due to mathematical complexity, it was very common in the past to adopt various assumptions in the formulation to ease the burden of computation. Common approaches adopted in the past may be categorized into the following three groups based on the ways the axial load in the rod is being formulated.

(1) The axial load is assumed to be dependent only on time and can be obtained by treating the rod as rigid [1-7]. This approach is also very popular in finite element formulations [8-11]. (2) The axial force is related to the transverse displacement by integrating the axial equilibrium equation [12-14]. Similar idea in the finite element approach can be found in [15-18]. (3) The third approach is to consider the effect of axial vibration, but assume that the axial force is proportional to the linear axial strain [19-21]. This approach has also been incorporated in finite element formulation [22].

In an effort to examine the validity of the above simplifications Chen and Chen [23] reformulated the equations of motion by assuming that the axial load is proportional to the Lagrangian strain. All the high order terms were retained in [23], and eigenfunction expansion method was used to facilitate numerical simulations. It is found that all the previous formulations cited above [1-22] give satisfactory results only when the crank speed is low. On the other hand when the crank speed is comparable to the first bending natural frequency of the connecting rod, all these simpli-

Contributed by the Mechanisms and Robotics Committee for publication in the JOURNAL OF MECHANICAL DESIGN. Manuscript received March 2002; rev. April 2003. Associate Editor: S. K. Agrawal. fied formulations overestimate the dynamic response by almost ten folds because terms of significant order of magnitude are removed inadequately. Chen and Huang [24] confirmed the findings in [23] by adopting a nonlinear finite element formulation taking into account all the high order terms in the strain energy function, including the usually-neglected quartic term of the displacement gradient.

The equations of motion derived in [23] and [24] are so complicated that it is impossible to perform any analytical study. Consequently, important physical insight and meaningful conclusions can be obtained only through large amount of calculations with wide range of parameters. In this paper we present an effort to simplify the equations of motion to a tractable form in a systematic manner and identify the important terms contributing to the dynamic behaviors which were usually ignored before. We first use the extended Hamilton's principle to reformulate the complete equations of motion of a damped connecting rod involving coupled axial and lateral vibrations. Rotary inertia effect will be included automatically. Eigenfunction expansion method is then adopted to discretize the partial differential equations. By appropriately ordering the magnitude of each term the coupled equations of motion can be simplified to a single one in terms of the transverse deflection. Based on this simplified equation multiple scale method can be used to derive the closed-form approximation of the steady state vibrations and the associated bifurcation set.

\section{Equations of Motion}

Figure 1 shows a slider-crank mechanism. The rigid crank of length $a$ rotates with constant speed $\Omega$. The length, cross section area, mass density, and Young's modulus of the connecting rod are $L, A, \rho$, and $E$, respectively. The mass of the slider is $m_{s} . X O Y$ is an inertial frame with its origin attached at the center of the rotating crank. $x A y$ is a moving frame with $x$-axis passing through the two ends of the connecting rod before deformation. $u(x, t)$ and 


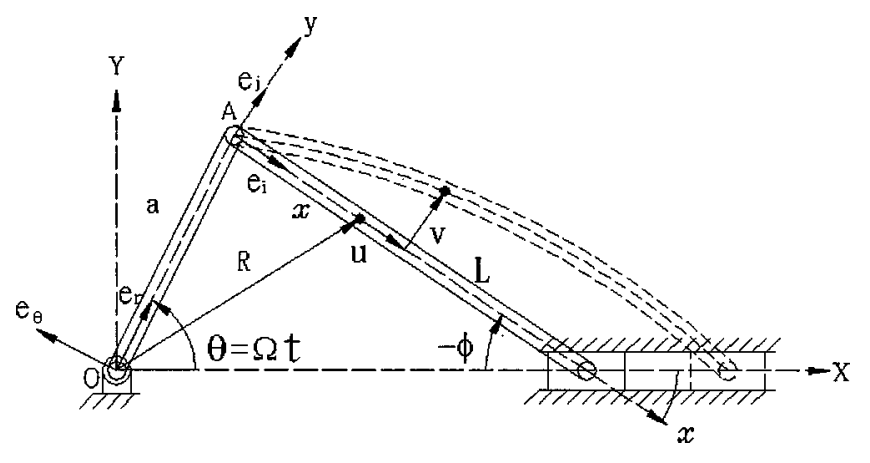

Fig. 1 Schematic diagram of a slider-crank mechanism

$v(x, t)$ denote the axial and transverse deflections of the connecting rod with respect to frame $x A y$. The position vector $\mathbf{R}(x, y, t)$ of a point in the connecting rod measured from the pivot $\mathrm{O}$ can be written as

$$
\mathbf{R}=a \mathbf{e}_{r}+\left(x+u-y v_{, x}\right) \mathbf{e}_{i}+(y+v) \mathbf{e}_{j}
$$

where $\left(\mathbf{e}_{r}, \mathbf{e}_{\theta}\right)$ and $\left(\mathbf{e}_{i}, \mathbf{e}_{j}\right)$ are rotating base vectors attached to points $\mathrm{O}$ and $\mathrm{A}$, respectively. The kinetic energy $T_{c}$ of the connecting rod is

$$
T_{c}=\frac{A}{2} \int_{0}^{L} \rho \dot{\mathbf{R}} \cdot \dot{\mathbf{R}} d x
$$

where

$$
\begin{aligned}
\dot{\mathbf{R}}(x, y, t)= & {\left[-a \Omega \sin (\theta-\phi)+u_{, t}-y v_{, x t}-\dot{\phi}(y+v)\right] \mathbf{e}_{i} } \\
& +\left[a \Omega \cos (\theta-\phi)+v_{, t}+\dot{\phi}\left(x+u-y v_{, x}\right)\right] \mathbf{e}_{j}
\end{aligned}
$$

$\theta=\Omega t$ is the rotation angle of the crank. $\phi$ is the angle between the $x$ - and $X$-axes.

$$
\phi=\sin ^{-1}\left(-\frac{a \sin \Omega t}{L}\right)
$$

It is noted that Eq. (2) includes the rotary inertia of the rod. The kinetic energy of the slider $T_{s}$ is

$$
T_{s}=\frac{m_{s}}{2} \dot{\mathbf{R}}(L, 0, t) \cdot \dot{\mathbf{R}}(L, 0, t)
$$

In this paper Euler-Bernoulli beam model is adopted and the axial strain at a point $(x, y)$ in the connecting rod can be written as

$$
e_{x x}=u_{, x}+\frac{1}{2} v_{, x}^{2}-y v_{, x x}
$$

Following a volume integral the strain energy $U_{c}$ of the connecting rod can be written as

$$
U_{c}=\frac{1}{2} \int_{0}^{L}\left[E A\left(u_{, x}^{2}+u_{, x} v_{, x}^{2}+\frac{1}{4} v_{, x}^{4}\right)+E I v_{, x x}^{2}\right] d x
$$

$I$ is the area moment of inertia of the connecting rod,

$$
I=A r^{2}
$$

$r$ is the radius of gyration of the cross section. Regarding the damping mechanism we assume that the connecting rod is made of a Kelvin-type viscoelastic material and the dissipation force is proportional to the strain rate. The variation of work done by this nonconservative internal force is

$$
\delta W=-\mu e_{x x, t} \delta e_{x x}
$$

$\mu$ is the material damping constant. By applying Hamilton's principle

$$
\int_{t_{1}}^{t_{2}}\left(\delta T_{c}+\delta T_{s}-\delta U_{c}+\delta W\right) d t=0,
$$

the equations of motion of the connecting rod can be obtained as

$$
\begin{aligned}
u_{, t t}- & \dot{\phi}^{2} u-\frac{E}{\rho}\left(u_{, x x}+v_{, x} v_{, x x}\right)-2 \dot{\phi}_{, t}-\ddot{\phi} v-x \dot{\phi}^{2} \\
& -a \Omega^{2} \cos (\Omega t-\phi)-\frac{\mu}{\rho}\left(u_{, x x t}+v_{, x t} v_{, x x}+v_{, x} v_{, x x t}\right)=0 \\
v_{, t t}- & \dot{\phi}^{2} v+\frac{E I}{\rho A} v_{, x x x x}+2 \dot{\phi} u_{, t}+\ddot{\phi} u \\
& -\frac{E}{\rho}\left(u_{, x x} v_{, x}+u_{, x} v_{, x x}+\frac{3}{2} v_{, x}^{2} v_{, x x}\right)+\frac{\mu I}{\rho A} v_{, x x x x t} \\
& -\frac{\mu}{\rho}\left(u_{, x x t} v_{, x}+2 v_{, x x} v_{, x t} v_{, x}+u_{, x t} v_{, x x}+v_{, x}^{2} v_{, x x t}\right)+x \ddot{\phi} \\
& -a \Omega^{2} \sin (\Omega t-\phi)+\frac{I}{A}\left(-\dot{\phi}^{2} v_{, x x}+v_{, x x t t}\right)=0
\end{aligned}
$$

There are two differences in Eqs. (10) and (11) above and Eqs. (7) and (8) in [23]. First of all, there is no damping mechanism in Eqs. (7) and (8) in [23]. Secondly, The last term in Eq. (11) above is due to rotary inertia of the connecting rod, which is ignored in [23].

The connecting rod is assumed to be simply-supported at both ends. After imposing the geometric constraint for the slider motion, i.e.,

$$
v(L, t)=-u(L, t) \tan \phi
$$

and following the variation procedure (9) the boundary conditions for $u$ and $v$ can be derived as

$$
\begin{gathered}
u(0, t)=v(0, t)=v_{, x x}(0, t)=v_{, x x}(L, t)=0 \\
{\left[\left(E A e_{x x}+\mu A e_{x x, t}\right)\left(1-v_{, x} \tan \phi\right)-I \tan \phi\left(E A v_{, x x x}\right.\right.} \\
\left.\left.+\mu A v_{, x x x t}\right)\right]_{x=L}=-m_{s} a_{X} / \cos \phi
\end{gathered}
$$

$a_{X}$ is the acceleration of the slider,

$$
a_{X}=-a \Omega^{2} \cos \Omega t-L \dot{\phi}^{2} \cos \phi+a \ddot{\phi} \sin \Omega t
$$

Boundary condition (14) has also been derived by Fung et al. [25], except that the important terms involving $v_{, x x x}$ are missing. It is noted that $v(L, t)$ is not exactly zero in general. However, it has been examined by Huang [26] with finite element method that imposing $v(L, t)=0$ only induces negligible local error for a crank length $a<0.2 L$. In this paper we focus on the case with crank length in this range. For such a case the geometric constraint (12) and the boundary condition (14) can be approximated as

$$
\begin{gathered}
v(L, t)=0 \\
{\left[E A e_{x x}+\mu A e_{x x, t}\right]_{x=L}=-\frac{1}{\cos \phi}\left[\frac{1}{2} \rho A L a \Omega^{2} \sin \phi \sin (\Omega t-\phi)\right.} \\
\left.-\frac{1}{3} \rho A L^{2} \ddot{\phi} \sin \phi+m_{s} a_{X}\right]
\end{gathered}
$$

\section{Nondimensionalization}

Equations (10) and (11) can be nondimensionalized by introducing the following dimensionless quantities,

$$
x^{*}=\frac{x}{L}, \quad a^{*}=\frac{a}{L}, \quad u^{*}=\frac{L}{r^{2}} u, \quad v^{*}=\frac{v}{r}, \quad \varepsilon=\frac{r}{L},
$$

$m_{s}^{*}=\frac{m_{s}}{\rho A L}, \quad P^{*}=\frac{P}{E A}, \quad \Omega^{*}=\frac{\Omega}{\omega_{b}}, \quad t^{*}=\omega_{b} t, \quad \mu^{*}=\frac{\mu r^{2} \pi^{4}}{\rho \omega_{b} L^{4}}$ 
$\omega_{b}$ is the lowest bending natural frequency of the connecting rod. It is noted that the above nondimensionalization relations are chosen so that both $u^{*}$ and $v^{*}$ are of order one when $\Omega^{*}$ is close to 1 [23]. After nondimensionalizing Eqs. (10) and (11) and assuming a one-mode approximation for $u^{*}$ and $v^{*}$ as following,

$$
\begin{aligned}
& u^{*}\left(x^{*}, t^{*}\right)=f\left(t^{*}\right) \sin \frac{\pi x^{*}}{2} \\
& v^{*}\left(x^{*}, t^{*}\right)=g\left(t^{*}\right) \sin \pi x^{*}
\end{aligned}
$$

we can obtain the equations for $f$ and $g$ as,

$$
\begin{aligned}
\varepsilon^{2}\left(\ddot{f}-\dot{\phi}^{2} f\right)-\varepsilon \frac{8}{3 \pi}(2 \dot{\phi} \dot{g}+\ddot{\phi} g) \\
\quad+\frac{1}{\pi^{2}}\left(\frac{f}{4}+\frac{7}{15} g^{2}\right)+\frac{\mu}{\pi^{2}}\left(\frac{\dot{f}}{4}+\frac{14}{15} g \dot{g}\right) \\
=\frac{8}{\pi^{2}} \dot{\phi}^{2}+\frac{4}{\pi} a \Omega^{2} \cos (\Omega t-\phi)-\frac{2}{\cos \phi}\left(\Theta-m_{s} a \Omega^{2} \cos \Omega t\right)
\end{aligned}
$$

$$
\begin{aligned}
& \varepsilon^{3} \frac{\pi^{2}}{2}\left(-\dot{\phi}^{2} g+\ddot{g}\right)+\varepsilon^{2} \frac{8}{3 \pi}(2 \dot{\phi} \dot{f}+\ddot{\phi} f) \\
& +\varepsilon\left(\ddot{g}-\dot{\phi}^{2} g+g+\frac{14}{15 \pi^{2}} f g+\frac{3}{8} g^{3}\right) \\
& +\varepsilon \mu\left(\dot{g}+\frac{14}{15 \pi^{2}} \dot{f} g+\frac{3}{4} g^{2} \dot{g}\right) \\
& =-\frac{2}{\pi} \ddot{\phi}+\frac{4}{\pi} a \Omega^{2} \sin (\Omega t-\phi)
\end{aligned}
$$

where

$$
\begin{aligned}
\Theta= & \frac{a}{3} \ddot{\phi} \sin \Omega t-\frac{1}{2} a^{2} \Omega^{2} \sin \Omega t \sin (\Omega t-\phi) \\
& +m_{s}\left(-\dot{\phi}^{2} \cos \phi+a \ddot{\phi} \sin \Omega t\right)
\end{aligned}
$$

All the asterisks in Eqs. (20) and (21) have been dropped for simplicity. The detail of the above discretization procedure can be found in [23]. It is noted that by this scaling method the slenderness ratio parameter $\varepsilon$ arises naturally and can be used to compare the order of magnitude of each term in the dimensionless equations. For instance, the effect of rotary inertia is of order $\varepsilon^{3}$ in Eq. (21) and can be ignored when $\varepsilon$ is small. Equations (20) and (21) can be solved numerically by Runge-Kutta method.

\section{Duffing Equation}

It is possible to reduce Eqs. (20) and (21) to a single equation if the order of magnitude of each term is examined carefully, especially in the high-speed range. By ignoring the $\varepsilon^{2}$ - and $\varepsilon$-terms and the terms containing $\mu \dot{f}$ in Eq. (20), and assuming $a$ to be small, we can express $f$ in terms of $g$ as

$$
f=-\frac{28}{15} g^{2}-\mu \frac{56}{15} g \dot{g}+8 a \pi \Omega^{2}\left(\pi m_{s}+2\right) \cos \Omega t
$$

After substituting Eq. (22) into Eq. (21), ignoring the $\varepsilon^{2}$ and $\varepsilon^{3}$-terms and the term containing $\varepsilon \mu \dot{f} g$, we obtain the single equation for $g$,

$$
\begin{aligned}
\ddot{g}+g & +2 \hat{\mu}_{1} \dot{g}+2 \hat{\mu}_{2} \dot{g} g^{2}+2 \hat{\kappa} \cos \Omega t g+\hat{\alpha} g^{3} \\
& =2 \hat{F}_{1} \sin \Omega t+2 \hat{F}_{2} \sin 2 \Omega t
\end{aligned}
$$

where

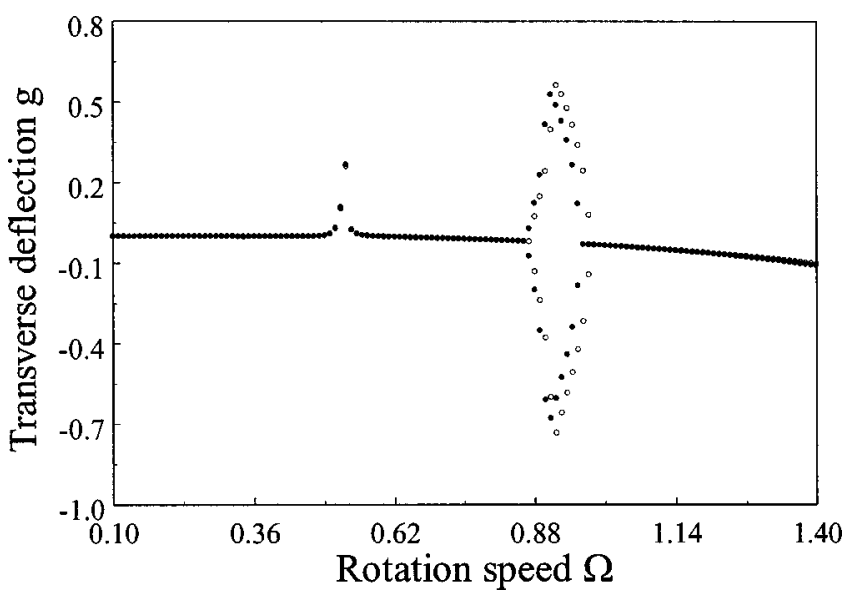

Fig. 2 Bifurcation diagram for parameters $\varepsilon=0.04, \mu=0.0146$, $m_{s}=0.5, a=0.05$. The black circles and the open circles represent the results from the two-equation approach and the single-equation approach, respectively.

$$
\begin{gathered}
\hat{\kappa}=\frac{56}{15} a \Omega^{2}\left(m_{s}+\frac{2}{\pi}\right), \quad \hat{\alpha}=\frac{3}{8}-\frac{392}{225 \pi^{2}}, \quad \hat{F}_{1}=\frac{a \Omega^{2}}{\varepsilon \pi}, \\
\hat{F}_{2}=a \hat{F}_{1}, \quad \hat{\mu}_{1}=\frac{\mu}{2}, \quad \hat{\mu}_{2}=\hat{\alpha} \mu
\end{gathered}
$$

It is noted that Eq. (23) is a damped Duffing equation under parametric and external excitations simultaneously. To test if this single equation is a good approximation to the original equations (20) and (21), we compare the steady state vibration predicted by Eq. (23) with the one predicted by Eqs. (20) and (21). We choose the following parameters for a slider-crank mechanism with relatively short crank: $\varepsilon=0.04, \mu=0.0146, m_{s}=0.5, a=0.05$. After specifying initial conditions for $f, \dot{f}, g, \dot{g}$ at certain crank speed $\Omega$, we examine the Poincare map of the response in the $g-\dot{g}$ space. The deflection is recorded when the crank is in the direction of the slider motion. If there exists periodic steady state solution with frequency $n \Omega$, the points in the Poincare map converge to $n$ separated points. We call this periodic steady state vibration a $\mathrm{P}-n$ solution. With damping constant $\mu=0.0146$ the steady state solution can be reached after 300 cycles. We record these steady state solutions in Fig. 2 for another 100 cycles. In other words, each point for a periodic solution in Fig. 2 actually represents 100 points at the same location in the Poincare map. The black circles and the open circles represent the results from the two-equation approach and the single-equation approach, respectively. It is observed that the results from the single equation match the results from two equations quite well for short crank length. In particular, we observe that the P-1 solutions bifurcate to P-2 at $\Omega=0.87$, and then bifurcate back to P-1 again at $\Omega=1$. In addition, we notice that there exists a spike around $\Omega=0.5$, which indicates a change of waveform in the solution. It is emphasized that for longer crank and large slenderness ratio the solution of the single equation may no longer be a good approximation to the one obtained by the original two equations. The effects of a long crank on the dynamic response of the connecting rod can be found in another paper by the same authors [29].

We plot the steady state solutions $g$ for three crank rotation cycles at three different rotation speeds, $\Omega=0.52,0.8$, and 0.95 in Figs. 3, 4, and 5, respectively. Other parameters are the same as those used in Fig. 2. The solid and dashed lines represent the solutions from two-equation and one-equation approaches, respectively. The chain lines in Figs. 3 and 4 are the solutions predicted by multiple scale method, which will be explained in the next section. Figure 3 shows that the response at $\Omega=0.52$ is a $\mathrm{P}-1$ 


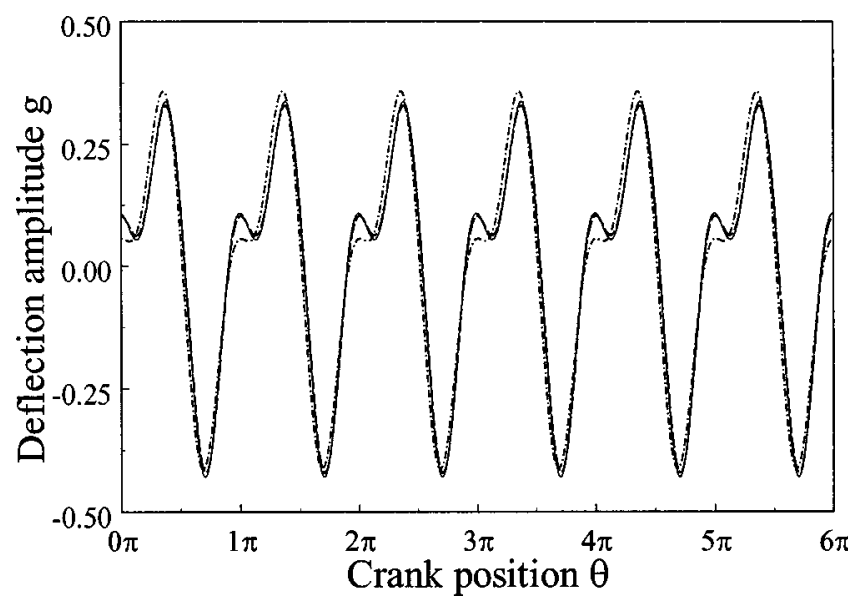

Fig. 3 Steady state vibration at $\Omega=0.52$. The solid and dashed lines represent the solutions from two-equation and oneequation approaches, respectively. The chain line is the solution predicted by multiple scale method.

solution consisting of at least two frequency components. Figure 4 shows a sinusoidal waveform at $\Omega=0.8$. Figure 5 shows that the response at $\Omega=0.95$ is a $\mathrm{P}-2$ solution.

\section{Multiple Scale Method}

After establishing the validity of Eq. (23) for the case with small slenderness ratio and crank length we can try to predict the steady state solution with multiple scale method. There are two rotation speeds at which we are particularly interested, i.e., $\Omega=1$ and 0.5 .

Case 1. Rotation speed near $\Omega=1$

In this case we rescale the coefficients in Eq. (23) by

$$
\begin{gathered}
\hat{\kappa}=\delta \kappa, \quad \hat{\alpha}=\delta^{2} \alpha, \quad \hat{F}_{1}=\delta^{2} F_{1}, \quad \hat{F}_{2}=\delta^{2} F_{2}, \\
\hat{\mu}_{1}=\delta^{2} \mu_{1}, \quad \hat{\mu}_{2}=\delta^{2} \mu_{2}
\end{gathered}
$$

$\delta$ is an artificial parameter introduced here to facilitate perturbation analysis. The proximity of $\Omega$ to unity can be expressed as [27]

$$
\Omega^{2}=1+\delta^{2} \sigma
$$

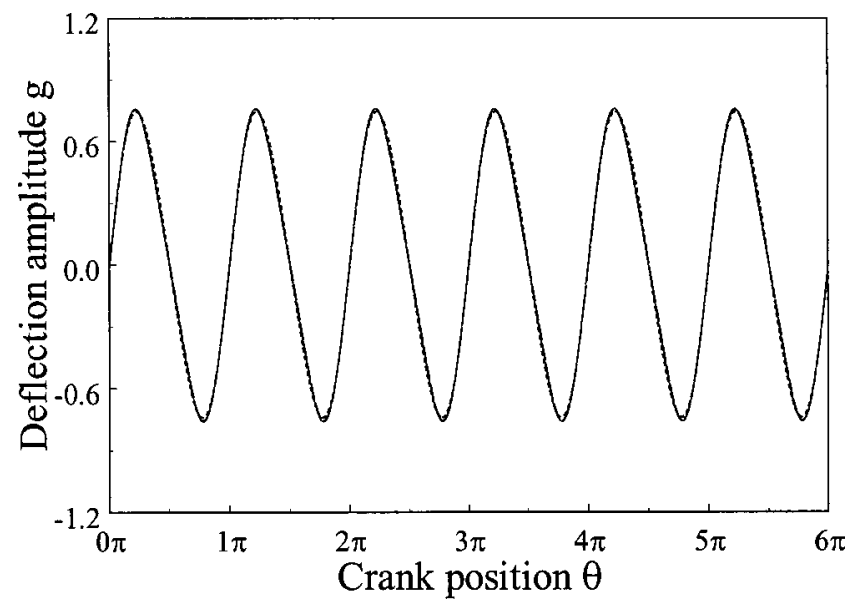

Fig. 4 Steady state vibration at $\Omega=0.8$. The solid and dashed lines represent the solutions from two-equation and oneequation approaches, respectively. The chain line is the solution predicted by multiple scale method.

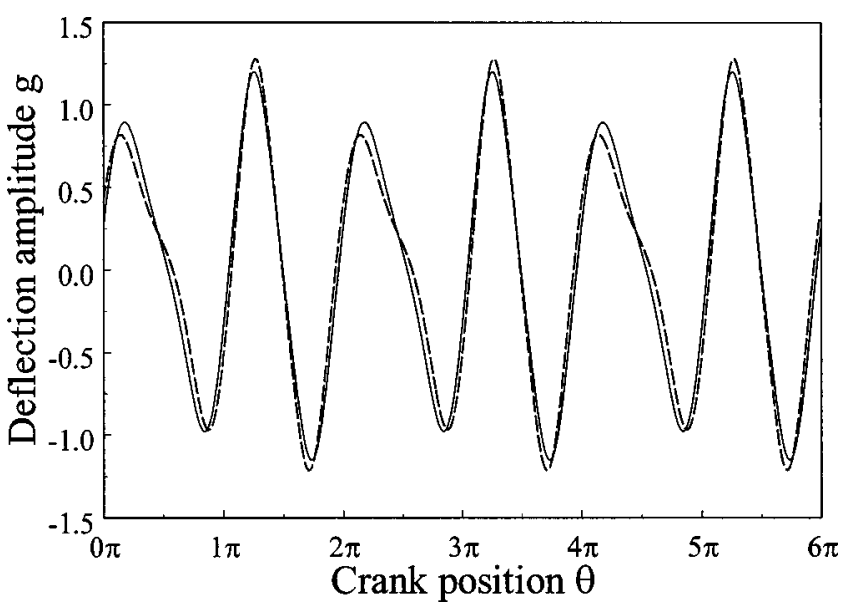

Fig. 5 A P-2 solution at $\Omega=0.95$. The solid and dashed lines represent the solutions from two-equation and one-equation approaches, respectively.

where $\sigma$ is a detunning parameter. After substituting Eqs. (25) and (26) into (23) we obtain

$$
\begin{aligned}
\ddot{g}+\Omega^{2} g= & -2 \delta \kappa \cos \Omega t g+\delta^{2}\left(\sigma g-2 \mu_{1} \dot{g}-2 \mu_{2} \dot{g} g^{2}-\alpha g^{3}\right. \\
& \left.+2 F_{1} \sin \Omega t+2 F_{2} \sin 2 \Omega t\right)
\end{aligned}
$$

Equation (23) can be recovered from (27) by specifying $\delta=1$. We assume the following expansion for $g$,

$$
\begin{aligned}
g(t, \delta)= & g_{0}\left(T_{0}, T_{1}, T_{2}\right)+\delta g_{1}\left(T_{0}, T_{1}, T_{2}\right)+\delta^{2} g_{2}\left(T_{0}, T_{1}, T_{2}\right) \\
& +\ldots
\end{aligned}
$$

where $T_{n}=\delta^{n} t$. Substituting Eq. (28) into Eq. (27) and equating coefficients of like powers of $\delta$ yields

$$
\begin{aligned}
& \delta^{0}: D_{0}^{2} g_{0}+\Omega^{2} g_{0}=0 \\
& \delta^{1}: D_{0}^{2} g_{1}+\Omega^{2} g_{1}=-2 D_{0} D_{1} g_{0}-2 \kappa \cos \Omega t g_{0} \\
& \delta^{2}: D_{0}^{2} g_{2}+\Omega^{2} g_{2}=-2 D_{0} D_{1} g_{1}-2 D_{0} D_{2} g_{0}-D_{1}^{2} g_{0}-2 \mu_{1} D_{0} g_{0} \\
& -2 \mu_{2} g_{0}^{2} D_{0} g_{0}-\alpha g_{0}^{3}-2 \kappa \cos \Omega T_{0} g_{1}+\sigma g_{0} \\
& +2 F_{1} \sin \Omega T_{0}+2 F_{2} \sin 2 \Omega T_{0}
\end{aligned}
$$

where $D_{n}=\partial / \partial T_{n}$. The general solution of Eq. (29) can be written as

$$
g_{0}=H\left(T_{1}, T_{2}\right) e^{i \Omega T_{0}}+\bar{H}\left(T_{1}, T_{2}\right) e^{-i \Omega T_{0}}
$$

where $\bar{H}$ is the complex conjugate of $H$. After substituting Eq. (32) into the right hand side of Eq. (30) we can rewrite

$$
D_{0}^{2} g_{1}+\Omega^{2} g_{1}=-2 i \Omega\left(D_{1} H\right) e^{i \Omega T_{0}}-H \kappa\left(1+e^{i 2 \Omega T_{0}}\right)+c c
$$

where $c c$ stands for the complex conjugates of the preceding terms. The secular term of Eq. (33) can be eliminated if

$$
D_{1} H=0
$$

The solution of Eq. (33) can then be written as

$$
g_{1}=\frac{H \kappa}{\Omega^{2}}\left(\frac{e^{i 2 \Omega T_{0}}}{3}-1\right)+c c
$$

After substituting Eqs. (32), (34), and (35) into Eq. (31) we can obtain the condition of eliminating the secular term of $g_{2}$ as 


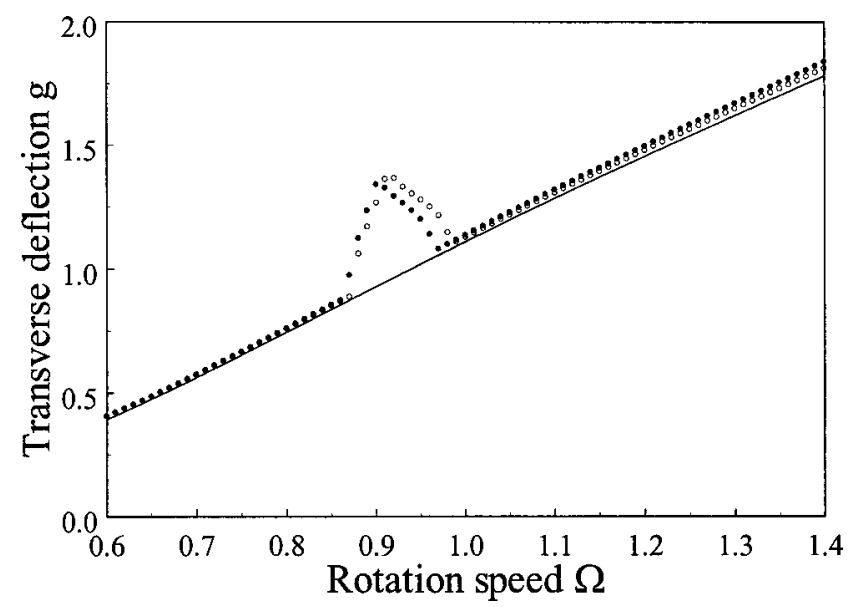

Fig. 6 Amplitude of steady state vibration as a function of rotation speed. Black and open circles are obtained by RungeKutta calculation with two- and one-equation approaches, respectively. The solid line is the solution predicted by multiple scale method.

$$
\begin{aligned}
& i 2 \Omega\left(D_{2} H+\mu_{1} H+\mu_{2} H^{2} \bar{H}\right)+3 \alpha H^{2} \bar{H}-\frac{\kappa^{2}}{\Omega^{2}}\left(\frac{2 H}{3}-\bar{H}\right)-\sigma H \\
& \quad+i F_{1}=0
\end{aligned}
$$

The complex function $H$ can be expressed as

$$
H=\frac{1}{2} h e^{i \beta}
$$

After substituting Eq. (37) into (36) we can conclude that steady state solutions $h$ and $\beta$ must satisfy the following equations,

$$
\begin{gathered}
2 \Omega^{3} \mu_{1} h+\frac{\Omega^{3} \mu_{2}}{2} h^{3}+2 \Omega^{2} F_{1} \cos \beta+\kappa^{2} h \sin 2 \beta=0 \\
\Omega^{2} \sigma h+\frac{2}{3} \kappa^{2} h-\frac{3}{4} \Omega^{2} \alpha h^{3}-2 \Omega^{2} F_{1} \sin \beta+\kappa^{2} h \cos 2 \beta=0
\end{gathered}
$$

After recovering the artificial parameter $\delta$ to 1 the steady state solution $g_{s}$ of Eq. (23) can be approximated as

$$
g_{s} \cong h\left[\cos (\Omega t+\beta)+\frac{\kappa}{3 \Omega^{2}}(\cos (2 \Omega t+\beta)-3 \cos \beta)\right]
$$

It is noted that the parameter $\kappa$ is proportional to $a$, and is small compared to 1 for the case with short crank. Therefore, it can be expected that the steady state response (40) is dominated by the term synchronous to the crank rotation. The solid line in Fig. 6 represents the amplitude of the steady state solution (40). The black circles are the results obtained by integrating Eqs. (20) and (21). The open circles represent the amplitude obtained by integrating Eq. (23). It is observed that the multiple scale solution approximates the Runge-Kutta solutions quite well, except in the range $0.87<\Omega<1$. For $\Omega=0.8$ the steady state waveform as predicted by Eq. (40) is plotted in Fig. 4 as the chain line.

It is noted that the multiple scale method fails to predict the P-2 solution in the range $0.87<\Omega<1$. However, the bifurcation speeds 0.87 and 1 may be verified by the Floquet theory. We express the solution $g$ of Eq. (23) as,

$$
g(t)=g_{s}+\hat{g}(t)
$$

where $g_{s}$ is given in Eq. (40) and $\hat{g}(t)$ is a small perturbation. After substituting Eq. (41) into Eq. (23), and linearizing with respect to $\hat{g}(t)$, we obtain the following equation,
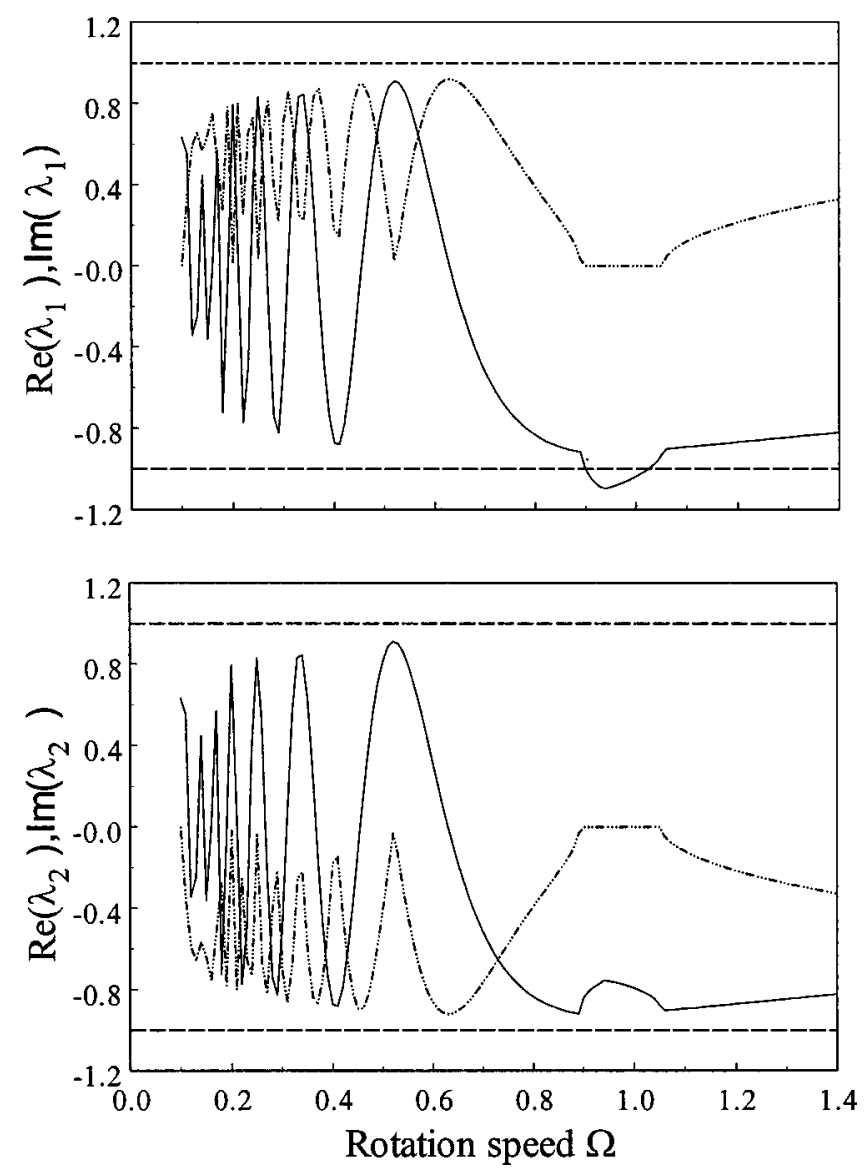

Fig. 7 Eigenvalues of the monodromy matrix. $\lambda_{1}$ leaves the unit circle at $\Omega=0.9$ and 1.02 , at which $\lambda_{1}=-1$.

$$
\ddot{\hat{g}}+\hat{g}+2 \kappa \cos \Omega t \hat{g}+\left(2 \mu_{1}+2 \mu_{2} g_{s}\right) \dot{\hat{g}}+\left(4 \mu_{2} g_{s} \dot{g}_{s}+3 \alpha g_{s}^{2}\right) \hat{g}=0
$$

Equation (42) is a damped Mathieu equation. After specifying two sets of initial conditions,

$$
\begin{array}{ll}
\hat{g}_{1}(0)=0, & \dot{\hat{g}}_{1}(0)=1 \\
\hat{g}_{2}(0)=1, & \hat{g}_{2}(0)=0
\end{array}
$$

and integrating Eq. (42) with Runge-Kutta method for one period $2 \pi / \Omega$ we obtain the solutions $\hat{g}_{1}(2 \pi / \Omega)$ and $\hat{g}_{2}(2 \pi / \Omega)$. The monodromy matrix [28] can then be obtained as,

$$
M=\left[\begin{array}{ll}
\hat{g}_{1}(2 \pi / \Omega) & \hat{g}_{2}(2 \pi / \Omega) \\
\dot{\hat{g}}_{1}(2 \pi / \Omega) & \dot{\hat{g}}_{2}(2 \pi / \Omega)
\end{array}\right]
$$

The eigenvalues $\lambda_{1}$ and $\lambda_{2}$ of matrix $M$ can then be calculated. The real part (solid line) and imaginary part (chain line) of $\lambda_{1}$ and $\lambda_{2}$ are plotted in Fig. 7. It can be observed that $\lambda_{1}$ leaves the unit circle at $\Omega=0.9$ and 1.02 , at which $\lambda_{1}=-1$. Therefore, the steady state solution undergoes period doubling at these two bifurcation speeds, as presented earlier in Fig. 2.

Case 2: Rotation speed near $\Omega=0.5$

In this case we have to rescale Eq. (23) by defining

$$
\hat{\kappa}=\delta \kappa, \quad \hat{\alpha}=\delta \alpha, \quad \begin{gathered}
\hat{F}_{1}=F_{1}, \quad \hat{F}_{2}=\delta F_{2}, \quad \hat{\mu}_{1}=\delta \mu_{1}, \\
\hat{\mu}_{2}=\delta^{2} \mu_{2}
\end{gathered}
$$

Equation (23) can then be rewritten as 


$$
\begin{aligned}
\ddot{g}+g= & 2 F_{1} \sin \Omega t+\delta\left(-2 \kappa \cos \Omega t g-2 \mu_{1} \dot{g}-\alpha g^{3}\right. \\
& \left.+2 F_{2} \sin 2 \Omega t\right)-\delta^{2} 2 \mu_{2} \dot{g} g^{2}
\end{aligned}
$$

By using expansion (28) and following the similar procedure as in case 1 , we obtain the following equations,

$$
\begin{aligned}
& \delta^{0}: D_{0}^{2} g_{0}+g_{0}=2 F_{1} \sin \Omega T_{0} \\
& \delta^{1}: D_{0}^{2} g_{1}+g_{1}=-2 D_{0} D_{1} g_{0}-2 \mu_{1} D_{0} g_{0}-\alpha g_{0}^{3}-2 \kappa \cos \Omega T_{0} g_{0} \\
&+2 F_{2} \sin 2 \Omega T_{0}
\end{aligned}
$$

The general solution of Eq. (48) is

$$
g_{0}=H e^{i T_{0}}+\frac{F_{1}}{i\left(1-\Omega^{2}\right)} e^{i \Omega T_{0}}+c c
$$

After substituting (50) into (49) and by defining

$$
2 \Omega=1+\delta \sigma
$$

we observe that the secular term of $g_{1}$ can be eliminated if

$$
\begin{gathered}
i 2\left(H^{\prime}+\mu_{1} H\right)+6 \alpha\left(\frac{F_{1}}{1-\Omega^{2}}\right)^{2} H+3 \alpha H^{2} \bar{H} \\
-i\left(\frac{F_{1} \kappa}{1-\Omega^{2}}-F_{2}\right) e^{i \sigma T_{1}}=0
\end{gathered}
$$

By substituting Eq. (37) into (52) and defining

$$
\gamma=\sigma T_{1}-\beta
$$

we obtain the equations for steady state solutions of $h$ and $\gamma$ as,

$$
\begin{gathered}
\mu_{1} h-\left(\frac{F_{1} \kappa}{1-\Omega^{2}}-F_{2}\right) \cos \gamma=0 \\
\sigma h-3 \alpha h\left(\frac{F_{1}}{1-\Omega^{2}}\right)^{2}-\frac{3 \alpha}{8} h^{3}-\left(\frac{F_{1} \kappa}{1-\Omega^{2}}-F_{2}\right) \sin \gamma=0
\end{gathered}
$$

After recovering the artificial parameter $\delta$ to 1 the steady state solution $g_{s}$ of Eq. (23) can be approximated as

$$
g_{s} \approx h \cos \left(2 \Omega T_{0}-\gamma\right)+\frac{2 F_{1}}{1-\Omega^{2}} \sin \Omega T_{0}
$$

It is noted that the second term in Eq. (56) is a particular solution and is proportional to $a / \varepsilon$, which is of order 1 in this paper. Therefore it can be expected that the steady state response (56) consists of two dominant frequency components. Equation (56) is traced as the chain line in Fig. 3 to compare with the Runge-Kutta solutions. The amplitudes of steady state solution (56) are plotted as solid lines in Fig. 8 for two different slider masses, $m_{s}=0$ and 0.3 . The open circles represent the amplitude obtained by integrating Eq. (23). It is observed that the solution predicted by multiple scale method matches the Runge-Kutta solution quite well for $m_{s}=0$. For $m_{s}=0.3$ while the multiple scale solution does not match the Runge-Kutta solution so well, it still captures the essence of the response near the peak.

\section{Conclusions}

A new formulation of the equations of motion of a flexible connecting rod is presented with emphasis on retaining all high order terms in the strain energy function, which are important especially in high-speed applications. The complete equations of motion involving both the axial and transverse vibrations are formulated by applying Hamilton's principle. The correct boundary conditions taking into account the slider-motion constraint are derived, which can be simplified to the conventional simplysupported boundary conditions when the crank is short. The par-

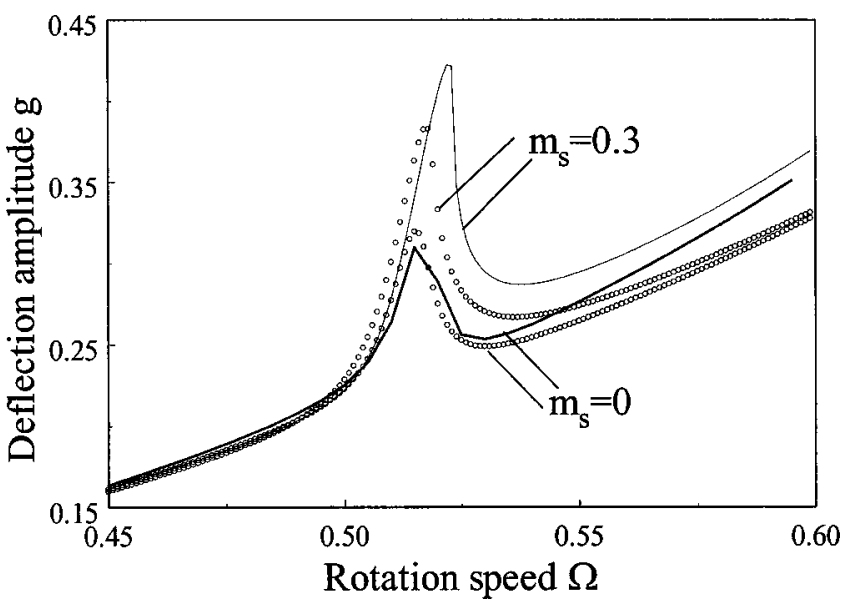

Fig. 8 Amplitudes of steady state vibration as a function of rotation speed in the neighborhood of $\Omega=0.5$. Open circles are obtained by Runge-Kutta calculation with one-equation approach. The solid lines are predicted by multiple scale method.

tial differential equations are discretized into two ordinary differential equations by a one-term eigenfunction expansion method. After carefully examining the order of magnitude of each term these two equations can be reduced to a single one, which turns out to be a Duffing equation under parametric and external excitations simultaneously. The nonlinear behavior of this equation is then studied by multiple scale method. Several conclusions can be summarized in the following.

1. The solution from the Duffing equation is a good approximation to the solution from the original two equations when the crank is short and the slenderness ratio of the connecting rod is small.

2. Combined effects of parametric and external excitations dominate the response when $\Omega$ is close to 0.5 and 1 . Away from these two speeds the response is dominated by the external excitation alone, and the solution is $\mathrm{P}-1$ with only one dominant frequency.

3. When the rotation speed $\Omega$ is close to 1 the response is a P-2 solution. The associated period doubling bifurcation speeds can be predicted by a Floquet theory.

4. In the neighborhood of $\Omega=0.5$ the response is a P-1 solution containing two dominant frequency components. The frequency response shows a peak near $\Omega=0.5$, whose height is found to be proportional to the slider mass.

\section{Acknowledgments}

The results presented here were obtained in the course of research supported by a grant from the National Science Council of the Republic of China.

\section{References}

[1] Neubauer, A. H., Cohen, R., and Hall, A. S., 1966, "An Analytical Study of the Dynamics of an Elastic Linkage," ASME J. Ind. Eng., 88, pp. 311-317.

[2] Badlani, M., and Kleninhenz, W., 1979, "Dynamic Stability of Elastic Mechanism," ASME J. Mech. Des., 101, pp. 149-153.

[3] Badlani, M., and Midha, A., 1982, "Member Initial Curvature Effects on the Elastic Slider-Crank Mechanism Response,” ASME J. Mech. Des., 104, pp. 159-167.

[4] Badlani, M., and Midha, A., 1983, "Effect of Internal Material Damping on the Dynamics of a Slider-Crank Mechanism," ASME J. Mech., Transm. Autom. Des., 105, pp. 452-459.

[5] Tadjbakhsh, I. G., 1982, "Stability of Motion of Elastic Planar Linkages With Application to Slider Crank Mechanism," ASME J. Mech. Des., 104, pp. 698 703.

[6] Tadjbakhsh, I. G., and Younis, C. J., 1986, "Dynamic Stability of the Flexible Connecting Rod of a Slider Crank Mechanism," ASME J. Mech., Transm., Autom. Des., 108, pp. 487-496. 
[7] Zhu, Z. G., and Chen, Y., 1983, "The Stability of the Motion of a Connecting Rod," ASME J. Mech., Transm., Autom. Des., 105, pp. 637-640.

[8] Bahgat, B. M., and Willmert, K. D., 1976, "Finite Element Vibrational Analysis of Planar Mechanisms," Mech. Mach. Theory, 11, pp. 47-71.

[9] Midha, A., Erdman, A. G., and Frohrib, D. A., 1978, "Finite Element Approach to Mathematical Modeling of High Speed Elastic Linkages," Mech. Mach. Theory, 13, pp. 603-618.

[10] Sunada, W., and Dubowsky, S., 1981, "The Application of Finite Element Methods to the Dynamic Analysis of Flexible Spatial and Co-Planar Linkage Systems," ASME J. Mech. Des., 103, pp. 643-651.

[11] Yang, Z., and Sadler, J. P., 1990, "Large-Displacement Finite Element Analysis of Flexible Linkages,” ASME J. Mech. Des., 112, pp. 175-182.

[12] Viscomi, B. V., and Ayre, R. S., 1971, "Nonlinear Dynamic Response of Elastic Slider-Crank Mechanism,” ASME J. Eng. Ind., 93, pp. 251-262.

[13] Hsieh, S. R., and Shaw, S. W., 1993, "Dynamic Stability and Nonlinear Resonance of a Flexible Connecting Rod: Continuous Parameter Model," Nonlinear Dyn., 4, pp. 573-603.

[14] Hsieh, S. R., and Shaw, S. W., 1994, "The Dynamic Stability and Nonlinear Resonance of a Flexible Connecting Rod: Single Mode Model," J. Sound Vib., 170, pp. 25-49.

[15] Nath, P. K., and Ghosh, A., 1980, "Kineto-Elastodynamic Analysis of Mechanisms by Finite Element Method," Mech. Mach. Theory, 15, pp. 179-197.

[16] Cleghorn, W. L., Fenton, R. G., and Tabarrok, B., 1981, "Finite Element Analysis of High-Speed Flexible Mechanisms," Mech. Mach. Theory, 16, pp. 407-424.

[17] Turcic, D. A., and Midha, A., 1984, "Dynamic Analysis of Elastic Mechanism System. Part I: Applications,” ASME J. Dyn. Syst., Meas., Control, 106, pp. 249-254.

[18] Thompson, B. S., and Sung, C. K., 1984, “A Variational Formulation for the Nonlinear Finite Element Analysis of Flexible Linkages: Theory, Implementation, and Experimental Results," ASME J. Mech., Transm., Autom. Des., 106, pp. $482-488$.
[19] Jasinski, P. W., Lee, H. C., and Sandor, G. N., 1970, "Stability and SteadyState Vibrations in a High Speed Slider-Crank Mechanism,” ASME J. Appl. Mech., 37, pp. 1069-1076.

[20] Jasinski, P. W., Lee, H. C., and Sandor, G. N., 1971, "Vibrations of Elastic Connecting Rod of a High Speed Slider-Crank Mechanism," ASME J. Ind. Eng., 93, pp. 636-644.

[21] Chu, S. C., and Pan, K. C., 1975, "Dynamic Response of a High Speed Slider-Crank Mechanism With an Elastic Connecting Rod,” ASME J. Ind. Eng., 97, pp. 542-550.

[22] Fung, R.-F., and Chen, H.-H., 1997, "Steady-State Response of the Flexible Connecting Rod of a Slider-Crank Mechanism With Time-Dependent Boundary Condition," J. Sound Vib., 199, pp. 237-251.

[23] Chen, J.-S., and Chen, K.-L., 2001, "The Role of Lagrangian Strain in the Dynamic Response of a Flexible Connecting Rod," ASME J. Mech. Des., 123, pp. $542-548$.

[24] Chen, J.-S., and Huang, C.-L., 2001, "Dynamic Analysis of Flexible SliderCrank Mechanisms With Nonlinear Finite Element Method," J. Sound Vib. 246, pp. 389-402.

[25] Fung, R.-F., Hwang, C.-C., and Chen, W.-P., 1997, “On the Non-Linear Dynamic Modeling of the Flexible Connecting Rod of a Slider-Crank Mechanism With Input Torque," J. Sound Vib., 203, pp. 523-532.

[26] Huang, C.-L., 2000, "Dynamic Analysis of Flexible Slider-Crank Mechanisms With Nonlinear Finite Element Method," Master Thesis, Department of Mechanical Engineering, National Taiwan University, Taipei, Taiwan.

[27] Nayfeh, A. H., and Sanchez, N. E., 1989, "Bifurcations in a Forced Softening Duffing Oscillator,” Int. J. Non-Linear Mech., 24, pp. 483-497.

[28] Nayfeh, A. H., and Balachandran, B., 1994, Applied Nonlinear Dynamics, John Wiley \& Sons, Inc., New York.

[29] Chen, J.-S., and Chian, C.-H., 2001, "Effects of Crank Length on the Dynamics Behavior of a Flexible Connecting Rod," ASME J. Vibr. Acoust., 123, pp. $318-323$. 